INPLASY

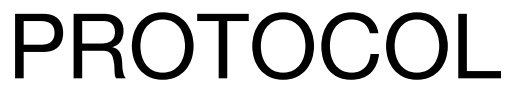

To cite: Zhu et al. Tuina therapy for the treatment of functional constipation in children and adolescents: A protocol for systematic review and metaanalysis. Inplasy protocol

202080073. doi:

10.37766/inplasy2020.8.0073

Received: 18 August 2020

Published: 18 August 2020

Corresponding author:

Ping Ma

3395820177@qq.com

Author Affiliation:

Chengdu University of TCM

Support: No. $\mathbf{3 0 8 7 3 2 2 4}$

Review Stage at time of this submission: The review has not yet started.

Conflicts of interest:

None.

\section{Tuina therapy for the treatment of functional constipation in children and adolescents: A protocol for systematic review and meta-analysis}

Zhu, K1; Ma, P2.

Review question / Objective: P: children and adolescents that are diagnosed with functional constipation. I: tuina therapy C: either chemotherapy or other types of intervention, such as acupuncture and herbal decoction. $O$ : alleviation in frequency of bowel movements, hard and/or large stools, painful defecation, fecal incontinence and abdominal pain.

Condition being studied: Functional constipation (FC) is one of the commonly seen functional bowel disorders (FBD) in clinical practice. FC affects patients' quality of life and imposes a waste of health care.

INPLASY registration number: This protocol was registered with the International Platform of Registered Systematic Review and Meta-Analysis Protocols (INPLASY) on 18 August 2020 and was last updated on 18 August 2020 (registration number INPLASY202080073).

\section{INTRODUCTION}

Review question / Objective: P: children and adolescents that are diagnosed with functional constipation. I: tuina therapy C: either chemotherapy or other types of intervention, such as acupuncture and herbal decoction. 0 : alleviation in frequency of bowel movements, hard and/ or large stools, painful defecation, fecal incontinence and abdominal pain.

Condition being studied: Functional constipation (FC) is one of the commonly seen functional bowel disorders (FBD) in clinical practice. FC affects patients' quality of life and imposes a waste of health care. 


\section{METHODS}

Participant or population: patients under the age of are diagnosed with functional constipation.

Intervention: Tuina therapy.

Comparator: Either chemotherapy or other types of intervention, except tuina therapy.

Study designs to be included: RCTs or quasi-RCTs.

Eligibility criteria: Children and adolescents that are diagnosed with FC.

Information sources: Electronic databases in both English and Chinese: Pubmed, Web of Science, Embase, Chinese Biomedical Literature Database (СВM), Chinese National Knowledge Infrastructure (CNKI), Wanfang Database and VIP China Science and Technology Journal Database (VIP) from their inception to 1 August, 2020, and the International Clinical Trials Registry Platform (ICTRP) and ClinicalTrials.gov.

Main outcome(s): Frequency of bowel movements, hard and/or large stools.

Quality assessment / Risk of bias analysis: Two investigators will independently evaluate methodological quality with the Review Manager (Revman V.5.3) developed by the Cochrane as a tool for detecting risk of bias (ROB). The quality of evidence will be evaluated under the guidance of Grading of Recommendations, Assessment, Development, and Evaluation (GRADE). We will assess them from the following aspects: random sequencing generation, allocation concealment, blinding of outcomes, completeness of outcomes and measurements, reporting bias and so on.

Strategy of data synthesis: We will depict a basic feature table of all included studies according to the participants, interventions, comparators, and outcomes (PICO) principle, which conveys general information, difference in methodology.
Subgroup analysis: When there are studies involved with high heterogeneity, we will perform a subgroup analysis to find out all possible explanation for the high heterogeneity. With subgroup analysis, we might come up with a clearer understanding of targeted population presenting to be more sensitive to the interventions.

Sensibility analysis: The stability of included data will be assessed by means of homogeneity exclusion. When there are studies of poor quality, we will conduct a sensitivity analysis with software Revman V.5.3.

Country(ies) involved: China.

Keywords: tuina therapy, functional constipation, children, Traditional Chinese Medicine, protocol.

Contributions of each author:

Author 1 - Kechun Zhu.

Author 2 - Ping Ma. 